\title{
Variations in the lipid composition of alfalfa roots during colonization with the arbuscular mycorrhizal fungus Glomus versiforme
}

Laura Gaspar

Instituto de Investigaciones Bioquímicas de La Plata (CONICET-UNLP), calles 60 y 120 La Plata (1900), Argentina; and Instituto de Botánica "Spegazzini" (UNLP), calle 53-477 La Plata (1900), Argentina

Ricardo Pollero

Instituto de Investigaciones Bioquimicas de La Plata (CONICET-UNLP), calles 60 y 120 La Plata (1900), Argentina

Marta Cabello

Instituto de Botánica "Spegazzini" (UNLP), calle 53-477 La Plata (1900), Argentina

Abstract: Lipids and their fatty acids were characterized and quantified during the development of the arbuscular mycorrhizal fungus Glomus versiforme in roots of alfalfa (Medicago sativa). A net increase in root total lipids was observed and was proportional to the development of arbuscular mycorrhizal fungus colonization. Triacylglycerols were the main lipid class in colonized roots and appear to have been synthesized by fungi. The increase of palmitoleic acid (characteristic of triacylglycerols from arbuscular mycorrhizal fungus) in roots colonized with $G$. versiforme reinforced this suggestion. Phosphatidylethanolamine and to a minor extent phosphatidylcholine also increased during the endophyte growth. Variations in the lipid classes of external mycelia isolated from one-, two- and three-mo old colonized roots with $G$. versiforme were also analyzed. Despite the continuous increase of triacylglycerols and phosphatidylethanolamine observed, the polar lipid class predominated in the external mycelia isolated from one-mo old colonized roots and the neutral lipid class prevailed in the external mycelia obtained from three-mo old mycorrhizal roots. Biosynthesis of triacylglycerols in intra- and extramatrical mycelia as well as an induced synthesis of polar lipids in roots are suggested as the consequence of fungi colonization.

Key Words: external mycelia, fatty acids, phospholipids, triacylglycerols

Accepted for publication August 1, 1996.

\section{INTRODUCTION}

Lipids are the most abundant compounds in arbuscular mycorrhizal fungi (AMF), although biochemical studies in these symbiont systems are scarce. There are available data on the lipid composition of few fungal species and only about some steps of the root colonization process (Beilby, 1980; Beilby and Kidby, 1980; Jabaji-Hare et al., 1984; Jabaji-Hare, 1988; Sancholle and Dalpé, 1993; Bentivenga and Morton, 1994).

Root colonization by AMF has both an internal and external phase. The development of structures within the root has been studied in detail (Smith and Walker, 1981; Bonfante-Fasolo, 1984) and the occurrence of lipid droplets in internal hyphae and vesicles has been reported (Mosse and Bowen, 1968; Ho and Trappe, 1973; Cox and Sanders, 1974; Cooper and Lösel, 1978; Nemec, 1981). Nevertheless, the characteristics of lipid classes and their quantitative variations during root colonization have not been determined yet. Little is known about the development of the external hyphae in relation to the internal phases of development (Abbott and Robson, 1984; Sylvia, 1992), and no research about the behavior of lipids during the development of extramatrical phase has been done. Then, neither the lipid composition during the development of external phase nor that of internal phase have been simultaneously and carefully analyzed in the same species. In order to obtain additional knowledge on the role of lipids in these biological systems and because the available information is so fragmentary, it is necessary to study lipids in the same species at different times of AMF colonization.

Glomus versiforme is characterized by a high lipid content in its spores (Gaspar et al., 1994a). We have also determined the variations in lipid and fatty acid composition in spores of $G$. versiforme at different times of germination (Gaspar et al., 1994b), observing that this process evoked a continuous decrease of triacylglycerols and an increase of phospholipids. In order to gain information on the behavior of lipids during the remaining colonization stages, the present work deals with the variations in lipid composition at the time of $G$. versiforme colonization in alfalfa roots, taking into account the internal phase as well as the external mycelia. 


\section{MATERIALS AND METHODS}

Isolation of mycorrhizal roots and external mycelia.Glomus versiforme (Karsten) Berch is the species of arbuscular mycorrhizal fungi (AMF) used in these experiments. The strain (LPS $\mathrm{N}^{\circ} 80$ ) was maintained on Medicago sativa L. (alfalfa). Plants were grown in $500 \mathrm{~mL}$ open pots of 9:1 sand : soil steamed mixture. Alfalfa seeds were sown in moistened sand, and after two wk the seedlings were transplanted to the pots and grown at room temperature. Plants were watered from below using a capillary system, and fed with a nutrient solution (Hewitt, 1952) lacking phosphate.

The AMF inoculum consisted of $5 \mathrm{~g}$ of rhizosphere soil from an alfalfa plant pot culture of an isolate of $G$. versiforme which contained spores, extramatrical mycelia and colonized root fragments. The AMF-uninoculated treatment (control) consisted of soil filtrate (Whatman No. 1 filter paper) from the rhizosphere of mycorrhizal plants containing common soil microorganisms, but no propagules of $G$. versiforme.

Plants were harvested after 30, 60, 90 and $120 \mathrm{~d}$. The roots were washed and rinsed three times with sterilized distilled water. The root system from each of three replicate groups of pots was divided to record a) AMF colonization, and b) lipid and fatty acid composition.

External mycelia were isolated from roots of 30-, 60-, and 90-d old alfalfa colonized with G. versiforme. The roots were washed and rinsed gently with sterilized water and the external mycelia collected with forceps under a dissecting microscope.

Mycorrhizal measurements.-The roots were cleared and stained (Phillips and Hayman, 1970), cut in 1-cm segments that were mixed and repeatedly subdivided to yield random samples of 30 root segments. Root samples were mounted on slides and examined under a compound microscope at $\times 160$ magnification. The percentage of total root length which was colonized with AMF, percentage of arbuscules, number of entry points, and vesicles were measured as described by Ocampo et al. (1980).

Extraction and analysis of lipids.-Inoculated and uninoculated roots, and external mycelia were frozen in liquid air and finely pulverized in a mortar. Total lipids of the resulting powder were extracted with chloroform/methanol $(2: 1 \mathrm{vol} / \mathrm{vol})$ by the method of Folch et al. (1957). Quantitative determination of the lipid classes was performed by TLC coupled to a flame ionization detector (FID) (Ackman, 1990) in a Iatroscan apparatus model TH 10 (Iatron Laboratories, Tokyo, Japan), after their separation on chromarods type S-III by the triple development procedure previously described in detail by Heras and Pol- lero, (1989). The signals from the FID were registered on a Hewlett-Packard (Palo Alto, CA) model HP-3396 A integrator.

Fatty acid analysis.-Aliquots of the extracts of total lipids were saponified with $10 \%$ potassium hydroxide in ethanol at $80 \mathrm{C}$ under nitrogen for $45 \mathrm{~min}$. The unsaponifiables were extracted with petroleum ether and discarded. The fatty acids were extracted with petroleum ether after acidification and esterified with $10 \%$ boron trifluoride in methanol. Fatty acid methyl esters (FAME) were analyzed by gas-liquid chromatography (GLC) using a Shimadzu model GC-9A apparatus fitted with a $30 \mathrm{~m}$ DB-23 phase J y W Scientific capillary column and a FID. Temperature was programmed for a linear increase of $3 \mathrm{C}$ per min from 160 to $220 \mathrm{C}$. Nitrogen was used as carrier gas at a flow rate of $20 \mathrm{~mL} / \mathrm{min}$. In routine assays FAME were analyzed in a Hewlett-Packard 5840 A apparatus equipped with a FID. A column packed with $10 \%$ SP-2330 on Chromosorb WAW was used. Temperature was programmed for a linear increase of 3 C per min from 140 to $220 \mathrm{C}$ (Pollero, 1986). The chromatographic peaks were identified as described earlier (Gaspar et al., 1994a).

\section{RESULTS}

Microscopy of stained roots showed that no fungi were present in uninoculated controls. Number of entry points and vesicles reached maxima values between 90 and 120 d (FIG. 1).

Results illustrated in Figs. 2 A and B show a comparatively high lipid concentration in roots when colonized by $G$. versiforme, neutral lipids being the main component. As seen in FIG. 2C, triacylglycerols were found to be the principal components of neutral lipids in mycorrhizal roots and they duplicated their concentration each mo of colonization. Free acids and free sterols were present in minor concentrations, but increased continuously (results not shown).

Total phospholipids showed an increase in both AMF-inoculated and uninoculated roots during the first mo of colonization (FIG. 3); this increase is more noticeable in the colonized roots between the first and the fourth mo of development. The variation in the total phospholipid fraction appeared to reflect an increase in phosphatidylethanolamine and to a lesser extent in phosphatidylcholine.

Percent compositions of the main fatty acids of alfalfa roots colonized with $G$. versiforme when compared to the percent values of the fatty acids of control roots are illustrated in FIG. 4. Palmitoleic acid (16:1) was the only fatty acid that increased significantly in roots when colonized by the endophyte. 

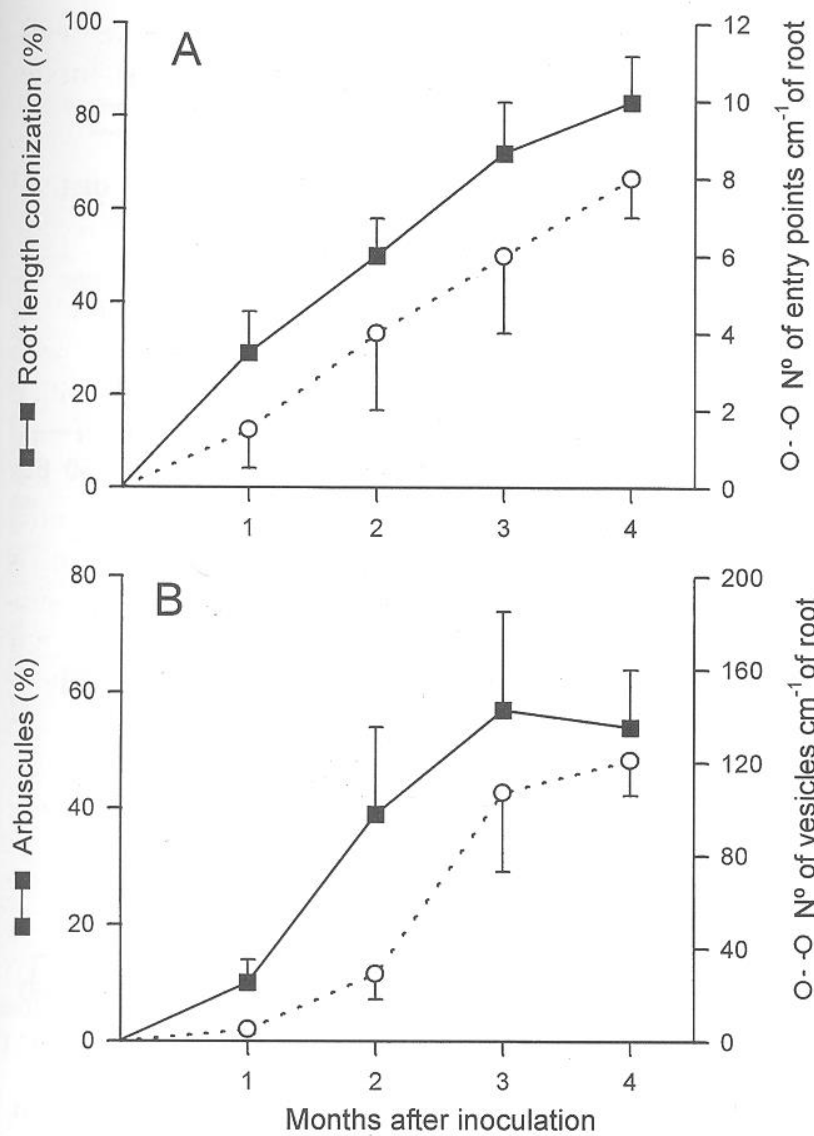

FIG. 1. Mycorrhizal measurements in alfalfa roots inoculated with Glomus versiforme after 1, 2, 3 and 4 mo. A: Percentage of root length colonization and number of entry points. B: Percentage of arbuscules and number of vesicles. Error bars $=$ SD.

The quantitative analysis of lipids from external mycelia isolated from alfalfa roots 30,60 , and $90 \mathrm{~d}$ after colonization by $G$. versiforme presented a progressive increase in total lipids, this increment being more evident in triacylglycerols than in phospholipids (FIG. 5). In the first mo of G. versiforme colonization, the proportion of phosphatidylethanolamine in external hyphae was higher than that of triacylglycerols. Nevertheless, in the second mo of colonization the amounts of triacylglycerols and phosphatidylethanolamine were similar. Then, in the third mo of colonization, triacylglycerols were found to be the principal lipid class in extramatrical mycelia.

\section{DISCUSSION}

The present study shows an increase in total alfalfa root lipids which is parallel to the degree of $G$. versiforme colonization. This variation in total lipids is the consequence of a significant increase in neutral lipids, principally triacylglycerols. As control roots
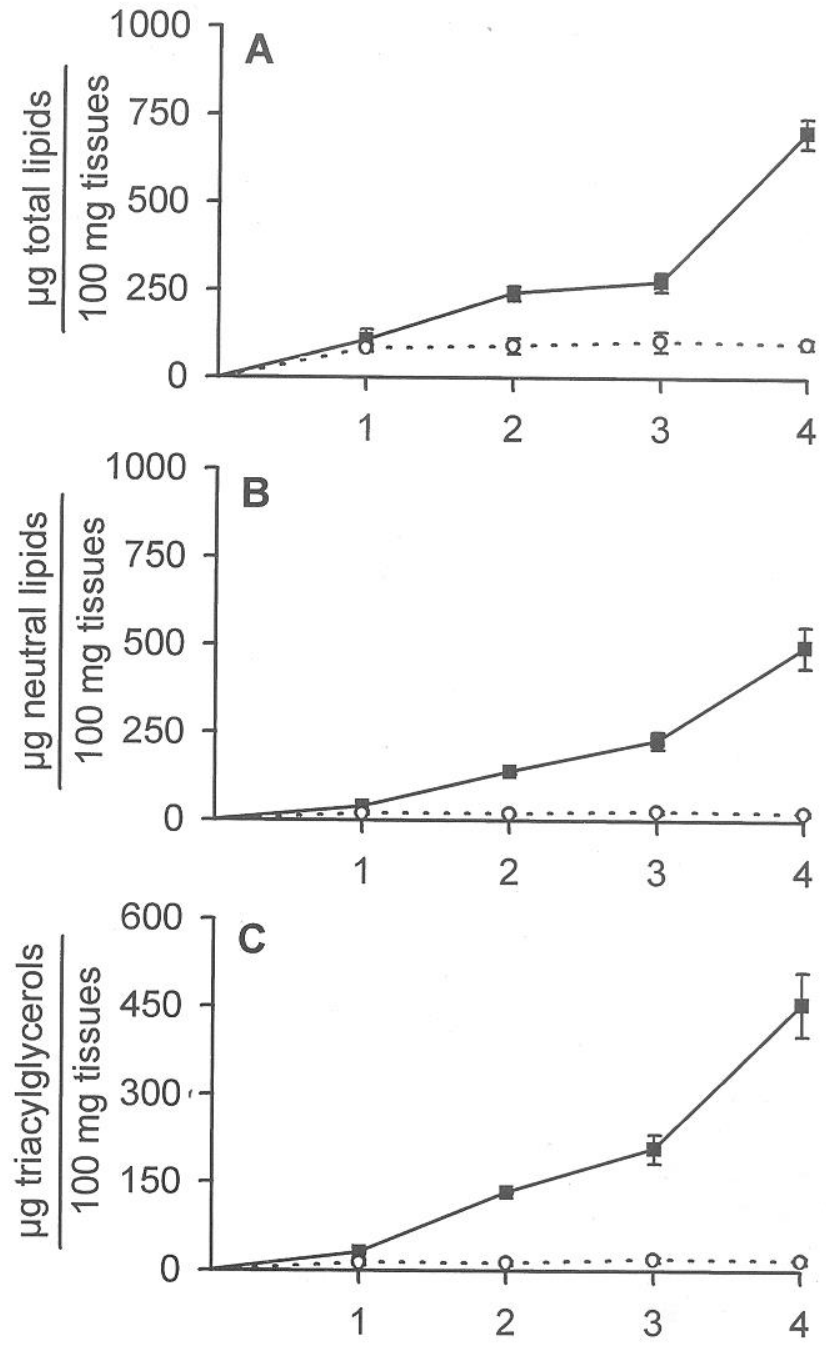

Months after inoculation

FIG. 2. Quantitative variations in lipids of alfalfa roots during the development of arbuscular mycorrhizal fungi. Lipids extracted from alfalfa roots at different stages of $G$. versiforme colonization, were identified and quantified by TLC-FID. Uninoculated roots were analyzed parallelly as control. A: Total lipids, B: Neutral lipids, C: Triacylglycerols. ( $\square---\square)$ Inoculated plants. $\left(\bigcirc_{-}-\bigcirc\right)$ Uninoculated plants. Average of three determinations \pm SD.

have not shown any increase in these lipid classes, it appears that triacylglycerols are mainly supplied by fungal hyphae. Thus, the triacylglycerol abundance, specially in the third and fourth mo of G. versiforme colonization, may be viewed as quantitative evidence of microscopic observations made by Cox et al. (1974) and Bonfante-Fasolo (1984) about the presence of lipid droplets in mature hyphae and vesicles. We have previously observed a scarce amount of triacylglycerols at advanced stages of spore germination (Gaspar et al., 1994b). This implies that the lipid sup- 

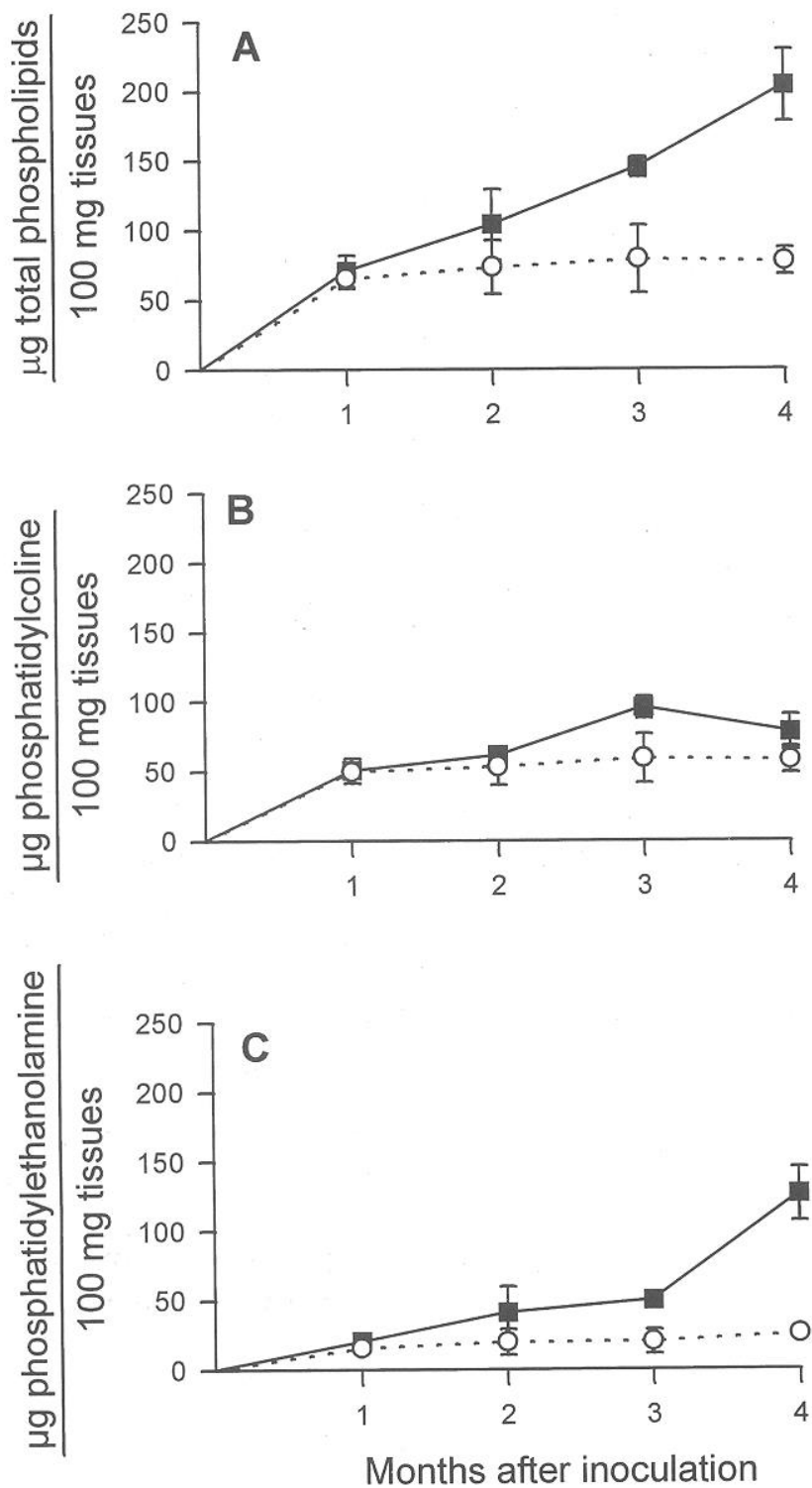

FIG. 3. Quantitative changes in total polar lipids and in concentrations of phospholipid classes in alfalfa roots during $G$. versiforme development. A: Total polar lipids, B: Phosphatidylcholine, C: Phosphatidylethanolamine. ( plants. Average of three determinations \pm SD.

ply to the root from spores as root colonization starts, would be negligible. In contrast, the continuous increase in triacylglycerols suggests an active synthesis of this lipid fraction when hyphae penetrate into the host cell and begin morphogenetic modifications of the whole root system. The low amount of triacylglycerols during the first mo of $G$. versiforme colonization may be attributed to the competition for photosynthate between both symbionts at early stages of AMF colonization. This competition can produce negative effects on plant growth which, in general, are temporary (Barea et al., 1984).
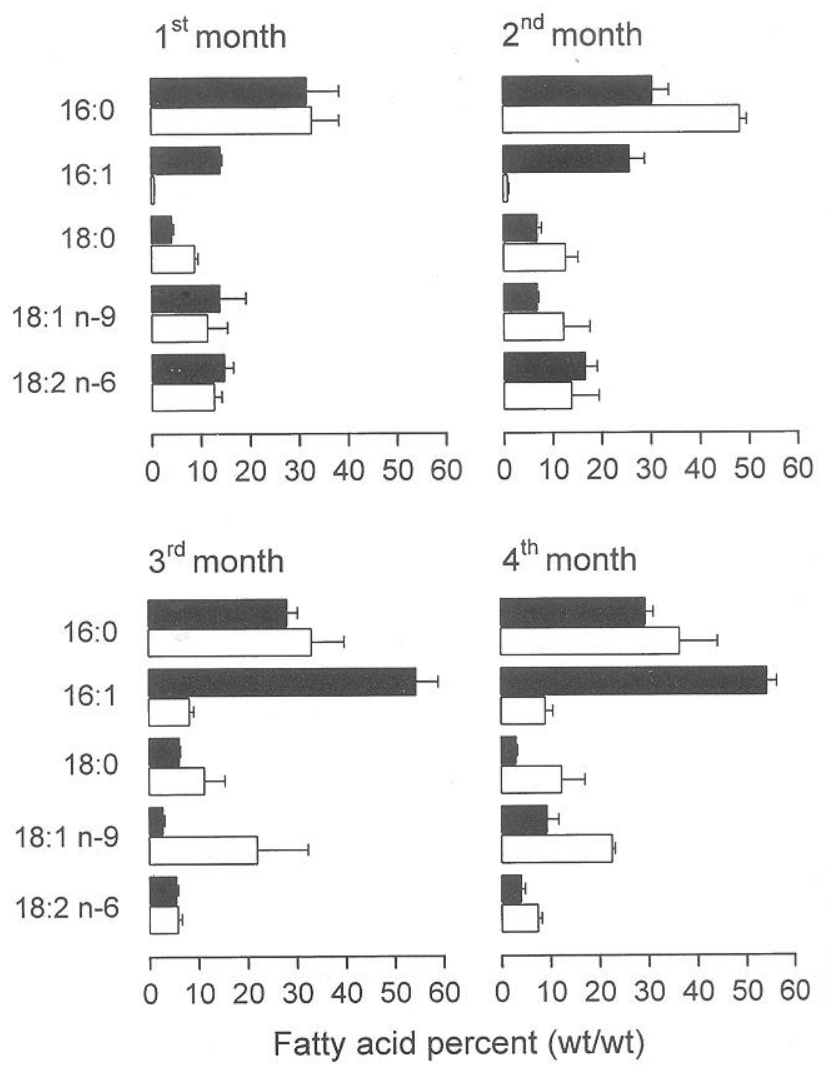

FIG. 4. Percentages of main fatty acids of alfalfa roots at different times of $G$. versiforme colonization. Root lipids from harvested plants were saponified; fatty acids were extracted and analyzed by GLC as methyl esters. Palmitic acid (16:0). Palmitoleic acid (16:1). Stearic acid (18:0). Oleic acid (18:1 n-9). Linoleic acid (18:2 n-6). Mycorrhizal plants. $\square$ Control plants. Average of four determinations \pm SD.

Smith and Gianinazzi-Pearson (1988) studied the possible determinants of an efficient symbiosis, and concluded that mycorrhizal plants seem to be less efficient during the early stages of the AMF colonization development. In contrast, they became more efficient at the advanced stages. One of these determinants is the biochemical sensitivity of the plant shown by a high photosynthetic rate. In turn, the storage of polysaccharides in roots only occurs concomitant with this high photosynthetic rate. Bevege et al. (1975) reported that radioactivity from ${ }^{14} \mathrm{CO}_{2}$ can be found in these storage compounds if photosynthesis is very high during and after labeling. This suggests that triacylglycerol accumulation in the fourth mo $G$. versiforme colonization would be not only the consequence of a high photosynthetic rate but also of a more efficient symbiosis during that period than at the beginning. Triacylglycerols should be another factor in determining symbiotic efficiency.

Phospholipids also increased their concentration 
DIZS Triacylglycerols

Free fatty acids

Phosphatidylethanolamine

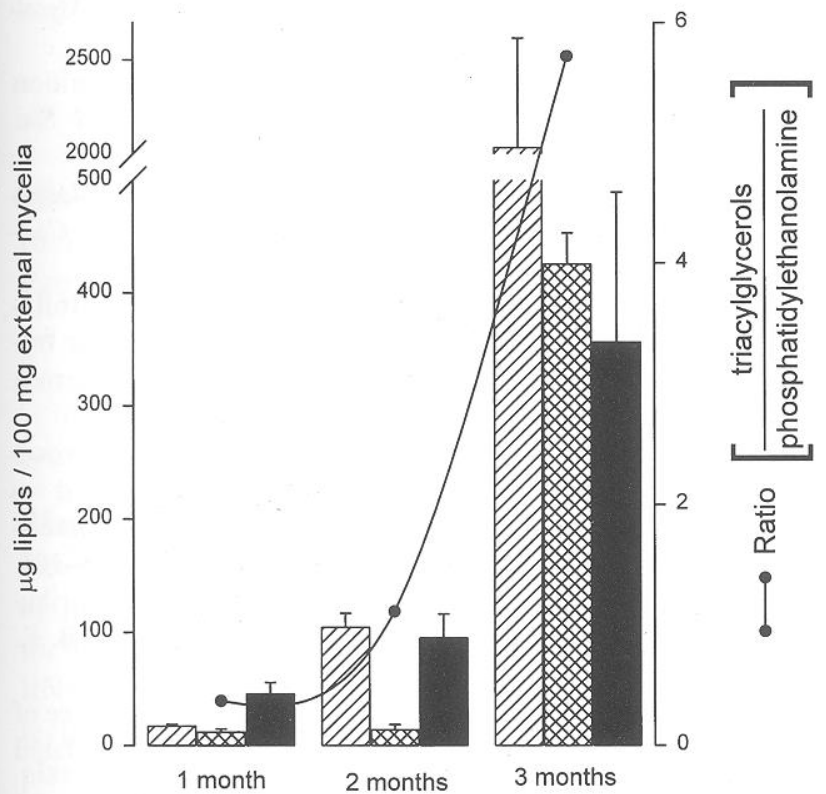

FIG. 5. Changes in lipid classes from external mycelia isolated from alfalfa roots colonized with $G$. versiforme after 1,2 and 3 mo. Average of three determinations. Bars $=\mathrm{SD}$.

in alfalfa roots during $G$. versiforme colonization, but not so markedly as the neutral lipids. The fact that phospholipids are major constituents of biological membranes would suggest that their increment is the result of the endophyte membrane synthesis. Free sterols, another membrane constituent, increase concomitantly, reinforcing the previous assumption. Taking into account that phosphatidylethanolamine is the most abundant phospholipid in $G$. versiforme spores (Gaspar et al., 1994a), the amount of this lipid class during AMF colonization would indicate active development of internal mycelia and vesicles. Since phosphatidylcholine was not detected in spores (Gaspar et al., 1994a) or in external mycelia of G. versiforme (results in this work), it seems unlikely that the fungi synthesize this phopholipid inside the root. Rather, the increase of phosphatidylcholine suggests fungal stimulation of synthesis by the root cells.

Palmitoleic acid seems to be a characteristic fatty acid of $G$. versiforme. It was determined in spores (Gaspar et al., 1994a) in which it reaches more than $50 \%$ of total fatty acids. In the present work similar percentages were found in colonized alfalfa roots while uninoculated plant roots only contained about one-sixth of this amount. As palmitoleic acid predominates in the triacylglycerol fraction, its content during colonization by $G$. versiforme seems to be an indicator of this lipid class accumulation.
During the development of the external mycelia, total lipid content increases reflecting an increase in triacylglycerol, phospholipid and free fatty acid levels. This variation runs in parallel with the above mentioned increment of triacylglycerols during root colonization. Without discarding the possible transference of these lipids from the internal to the external mycelia, our results seem to indicate that biosynthesis of lipids in extramatrical mycelia is important. Anyway, the carbons for the biosynthesis of these lipids by fungi would be provided by host metabolites. This fact implies that the catabolism of hexoses (or other compounds transferred) in the mycobiont would be important not only for the production of energy and reducing power, but also for providing carbon skeletons for their use in anabolism of triacylglycerols and other lipids. The detection of very little labeled sugars in external hyphae when plants photosynthesized in the presence of ${ }^{14} \mathrm{CO}_{2}$ allowed Bevege et al. (1975) to suppose that large quantities of the remaining labeled compounds would be lipids, lipoproteins, aminoacids and ketoacids. Our results indicate that these lipids are triacylglycerols and in a minor extent phospholipids and free acids. Future in vitro studies of the external mycelia capacity to biosynthesize lipids are indeed necessary.

\section{ACKNOWLEDGMENTS}

This research was supported by grants from CONICET, Argentina and Efamol Research Institute, Canada. R. Pollero and M. Cabello are members of Carrera del Investigador, CIC, Bs. As., Argentina.

\section{LITERATURE CITED}

Abbott, L. K., and A. D. Robson. 1984. Formation of external hyphae in soil by four species of vesicular-arbuscular mycorrhizal fungi. New Phytol. 99: 245-255.

Ackman, R. G., C. A. Mc Leod, and A. K. Banerjee. 1990. An Overview of Analyses by Chromarod-Iatroscan TLCFID. J.Planar Chromatogr. 3: 450-489.

Barea, J. M., C. Azcon-Aguilar, and B. Roldan-Fajardo. 1984. Avances recientes en el estudio de las micorrizas V-A. I Formación, funcionamiento y efectos en nutrición vegetal. Anal. Edafol. Agrobiol. 44: 659-677.

Beilby, J. P. 1980. Fatty acid and sterol composition of ungerminated spores of the vesicular-arbuscular mycorrhizal fungus, Acaulospora laevis. Lipids 15: 949-952.

—, and D. K. Kidby. 1980. Sterol composition of ungerminated and germinated spores of the vesicular arbuscular mycorrhizal fungus Glomus caledonius Lipids 15: 375-378.

Bentivenga, S. P., and J. B. Morton. 1994. Stability and heritability of fatty acid methyl ester profiles of glomalean endomycorrhizal fungi. Mycol. Res. 98: 1419-1426.

Bevege, D. I., G. D. Bowen, and M. F. Skinner. 1975. Com- 
parative carbohydrate physiology of ecto and endomycorrhizas. Pp. 149-174. In: Endomycorrhizas. Eds., F. E. Sanders, B. Mosse, and P. B. Tinker. Academic Press, London.

Bonfante-Fasolo, P. 1984. Anatomy and morphology of VA mycorrhizae. Pp. 5-32. In: VA Mycorrhiza. Eds., C. L1. Powell, and O. Bagyaraj. CRC Press, Boca Raton, Florida.

Cooper, K. M., and D. M. Lösel. 1978. Lipid physiology of vesicular arbuscular mycorrhiza. New Phytol. 80: 143151.

Cox, G., and F. E. Sanders. 1974. Ultrastructure of the host fungus interfase in a vesicular-arbuscular mycorrhiza. New Phytol. 73: 901-912.

Folch, J., M. Lees, and G. H. Sloane-Stanley. 1957. A simple method for the isolation and purification of total lipids from animal tissues. J. Biol. Chem. 226: 497-509.

Gaspar, M. L., R. J. Pollero, and M. N. Cabello. 1994a. Glomus antarcticum: the lipids and fatty acid composition. Mycotaxon 51: 129-136.

- - , and 1994b. Triacylglycerol consumption during spore germination of vesicular-arbuscular mycorrhizal fungi. J. Am. Oil Chem. Soc. 71: 449452.

Heras, H., and R. J. Pollero. 1989. Blood lipids of the small octopus O. tehuelchus (Mollusca Cephalopoda) at different stages of sexual maturation. Comp. Biochem. Physiol. 92A: 571-575.

Hewitt, E. J. 1952. Sand water culture methods used in the study of plant nutrition. Commonwealth Agriculture Bureau, Technical Communication No. 22. pp. 547.

Ho, I., and J. M. Trappe. 1973. Translocation of ${ }^{14} \mathrm{C}$ from Festuca plants to their endomycorrhizal fungi Nature 244: 30-31.

Jabaji-Hare, S., A. Deschene, and B. Kendrick. 1984. Lipid content and composition of vesicles of a vesicular-arbuscular mycorrhizal fungus. Mycologia 76: 1024-1030. . 1988. Lipid and fatty acid profiles of some vesicular-arbuscular fungi: contribution to taxonomy. Mycologia 80: 622-629.

Mosse, B., and G. D. Bowen. 1968. A key to the recognition of some Endogone spore types. Trans. Brit. Mycol. Soc. 51: 461-467.

Nemec, S. 1981. Histochemical characteristics of Glomus etunicatus infection of Citrus limon fibrous roots. Canad. J. Bot. 59: 609-617.

Ocampo, J. A., J. Martin, and D. S. Hayman. 1980. Influence of plant interactions on vesicular-arbuscular mycorrhizal infection. I Host and non-host plant grown together. New Phytol. 84: 27-35.

Phillips, J. M., and S. D. Hayman. 1970. Improved procedures for clearing roots and staining parasitic and vesicular-arbuscular mycorrhizal fungi for rapid assessment of infection. Trans. Brit. Mycol. Soc. 55: 158-161.

Pollero, R. J. 1986. Lipid and fatty acid seasonal distribution among tissues of Myriophyllum quitense $\mathrm{H}$. B. K. Aquatic Botany 26: 103-111.

Sancholle, M., and Y. Dalpé. 1993. Taxonomic relevance of fatty acids of arbuscular mycorrhizal fungi and related species. Mycotaxon 49: 187-193.

Smith, S. E., and N. A. Walker. 1981. A quantitative study of mycorrhizal infection in Trifolium: separate determination of the rates of infection and of mycelial growth. New Phytol. 89: 226-240.

, and V. Gianinazzi Pearson. 1988. Physiological interactions between symbionts in vesicular-arbuscular mycorrhizal plants. Ann. Rev. Plant Physiol. Plant Mol. Biol. 39: 21-244.

Sylvia, D. M. 1992. Quantification of external hyphae of vesicular-arbuscular mycorrhizal fungi. Pp. 53-65. In: Methods in Microbiology. Eds., J. R. Norris, D. J. Read, and A. K. Varma. Academic Press, New York. 